
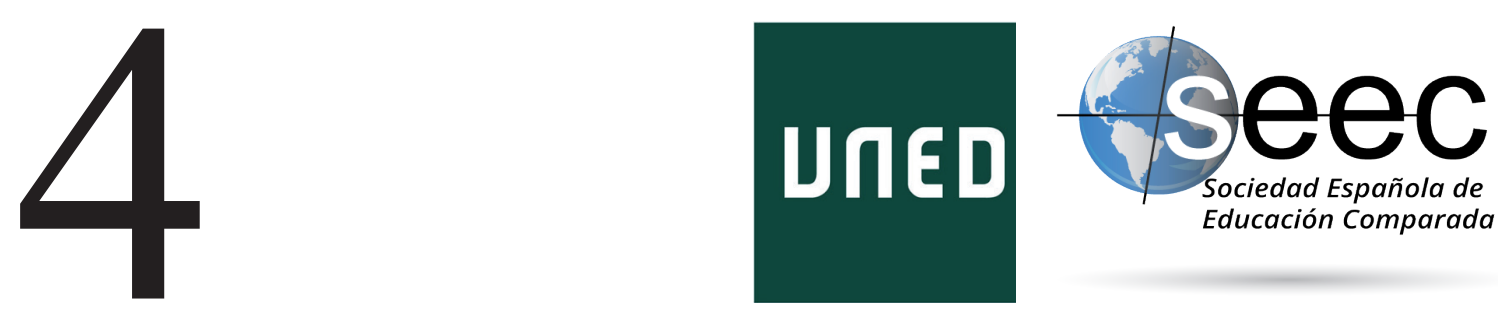

\title{
Gobernanza de la formación profesional dual española: entre la descoordinación y la falta de objetivos
}

Governance of the Spanish dual vocational training: between the lack of coordination and the lack of objectives

\section{Pablo Sanz de Miguel*}

DOI: $10.5944 /$ reec.30.2017.18705

Recibido: 24 de marzo de 2017 Aceptado: 18 de diciembre de 2017

\footnotetext{
* Pablo Sanz de Miguel: Doctor en Sociología por la Universidad Autónoma de Barcelona (UAB). Investigador en Notus y profesor asociado en en el Departametno de Sociología de la UAB. Datos de contacto: E-mail: pablo.sanz@notus-asr.org
} 


\title{
Resumen
}

La Formación Profesional (FP) dual está en la actualidad en fase de desarrollo en España, representándose como una opción política atractiva para facilitar la transición hacia el empleo de los jóvenes y adaptar sus competencias a los requerimientos productivos. En este contexto, la cuestión de sus estructuras de gobernanza suscita interés, dado que de estas depende, en buena parte, la eficacia y el éxito de un sistema que requiere una coordinación eficaz entre los distintos agentes con interés en la formación. Este artículo proporciona unas dimensiones de análisis para entender y clasificar los sistemas de gobernanza de la FP dual, analizando a partir de estas el incipiente sistema español, regulado en 2012, que se compara, a su vez, con los casos de dos países europeos, Dinamarca y Alemania, en los cuales la FP dual está institucionalizada desde hace décadas. Se trata, no obstante, de una comparación asimétrica dado que Dinamarca y Alemania se utilizan como elementos de contraste con el caso español, el cual es analizado con mayor profundidad recurriendo, además de a un análisis normativo, a un trabajo de campo basado en grupos de discusión. El artículo concluye evidenciando que el incipiente sistema español de FP dual actual tiene un amplio margen de mejora en el terreno de la gobernanza, requiriendo un marco legal más coherente y dotado de mayor consistencia, una mejora sustancial de la coordinación entre las distintas administraciones y actores involucrados, un reforzamiento del papel de los representantes de los trabajadores y una definición más precisa de los objetivos perseguidos.

Palabras clave: formación profesional dual; gobernanza; consistencia del marco legal; participación; racionalidad de la agencia

\begin{abstract}
Spain is currently developing a dual Vocational Education Training (VET), which is represented as an attractive political option to improve the transition of young people into the employment system and adapt their competences to the employers' demands. In this context, attention should be drawn to the issue of its governance structures. Governance structures ensure the efficiency and success of dual VET systems that require the coordination of activities of collective actors and the closer involvement of some agents such as companies. This article provides some analytical dimensions to understand and classify dual VET systems. Then it analyses through these dimensions the emergent dual VET system of Spain, which is compared with the cases of Denmark and Germany. It is, however, an asymmetric comparison, because Denmark and Germany are used as contrasting cases with the Spanish case, which is analysed more extensively through a normative analysis and a filed work based on focus groups. The article shows that there is room for improvement for the emerging Spanish dual vocational training system. It would require a more coherent and consistent legal framework, a substantial improvement in terms of coordination of the different administrations and actors involved, a reinforced role of employees' representatives and more precise definition of goals to be attained.
\end{abstract}

Key Words: dual vocational training; governance; Spain; legal framework consistency; participation; agency rationality 


\section{Introducción}

La Formación Profesional (FP en adelante) dual, entendida generalmente como un sistema en el cual la formación teórica en el centro educativo y la práctica en el centro de trabajo se alterna en cortos intervalos de tiempo, se ha situado en los últimos años en el centro del debate político español. En el actual contexto de elevado paro juvenil, esta se representa como una opción política atractiva para facilitar la transición hacia el empleo de los/las jóvenes o mejorar la adecuación de los contenidos formativos a las demandas empresariales. Este proceso ha sido, además, impulsado por iniciativas europeas tales como el Memorándum de Cooperación en materia de Formación Profesional, aprobado en Berlín el 11 de diciembre de 2012, o la Alianza Europea para la Formación de Aprendices, firmada el 2 de julio de 2013 por la Comisión Europea, la Presidencia del Consejo de la Unión Europea y las federaciones europeas de sindicatos y de organizaciones empresariales.

El sistema de FP inicial español no se considera como dual, según la terminología comúnmente empleada, sino como school based (Eichhorst, Rodríguez-Planas, Schmidl y Zimmermann, 2012), dado que la formación teórica y práctica en la empresa no se alterna. Esta última se lleva a cabo una vez finalizada la formación en el centro educativo, a través del denominado módulo de «Formación en el centro de trabajo» (FCT), que solo representa el $20 \%$ del total que los alumnos reciben a lo largo de dos cursos académicos.

El sistema de FP español ha sido analizado por diversos autores (Homs, 2008; Merino, 2009; Merino, García, Casal y Sánchez, 2011; Marhuenda, 2012; Planas, 2012; García, Casal, Merino y Sánchez, 2013). La literatura existente destaca el incremento continuado de jóvenes que optan por esta vía desde los años 80, como resultado del aumento del prestigio y la consideración social de una formación que, con la reforma educativa de 1990 (Ley Orgánica General del Sistema Educativo, LOGSE), se vio sujeta a un proceso de modernización y elevación de su nivel de calidad a través, entre otras medidas, de su equiparación con el bachillerato (Homs, 2008; Marhuenda, 2012). Gracias a esto, la FP tiende a representarse a día de hoy como más práctica y orientada al mercado laboral que otras iniciativas. En este sentido, los estudios sobre la inserción laboral de los jóvenes que cursan FP muestran que estos alcanzan tasas de inserción superiores. Frente estos hechos positivos, Sala, Planas y Van Rompaey (2010) exponen, recurriendo a datos de la a encuesta ETEFIL ${ }^{1}$, que tan solo un tercio de los matriculados en FP trabaja en «aquello para lo que se formaron», es decir, alcanza una correspondencia biyectiva entre empleos (definidos por especialidad y nivel) y titulaciones de FP. Esta realidad reforzaría una percepción ampliamente extendida, sobre todo entre el empresariado, que considera a la oferta formativa existente desligada e incluso ajena a las necesidades y requerimientos del sistema productivo (Lope y Alós, 2013). De estas perspectivas, se consideran por tanto insuficientes las vías que el sistema ofrece para conectar la formación impartida en los centros de FP a las demandas empresariales.

Sensible a las críticas que enfatizan la falta de adecuación de la oferta formativa del sistema de FP a los requerimientos productivos e impelido a buscar soluciones a un paro juvenil situado en torno al 50 \% en el año 2012, el gobierno del Partido Popular

1 La Encuesta de Transición Educativo-Formativa e Inserción Laboral (ETEFIL) está dirigida a jóvenes de educación secundaria y formación profesional (tanto del sistema educativo como ocupacional). $\mathrm{Su}$ objetivo principal es conocer las diferentes formas de transición desde la educación y la formación al mercado laboral, como apoyo a la toma de decisiones en materia de educación, formación profesional y empleo 
emprendió una reforma del sistema de FP, plasmada, fundamentalmente, en el Real Decreto 1529/2012. Dicho RD regula un sistema de FP dual que otorga mayor peso a la formación en la empresa. Así, se establece, entre otros aspectos que, en este modelo, al menos el 33 \% de la formación debe impartirse en una empresa, frente al $20 \%$ prevista en el modelo tradicional. Este nuevo sistema habrá de convivir con el sistema tradicional, disponiendo en el momento actual de una escasa implantación. Así, tan solo el $2 \%$ del total del alumnado de FP matriculado en el curso 2013-2014 lo estaba en régimen dual (SGOFP, 2014).

En este contexto, conviene prestar atención a las estructuras de gobernanza del incipiente sistema de FP dual. El término gobernanza ha sido estudiado desde diferentes ópticas y disciplinas, siendo especialmente relevantes los estudios politológicos centrados en la Unión Europea (Hooghe y Marks, 2001; Mariscal, 2003). El estudio de la gobernanza se centra en los mecanismos que, en sistemas sociales complejos, sirven para coordinar las actividades de diversas instituciones y actores, tanto públicos como privados, y emplazados en distintos niveles (transnacional, nacional o regional), que tienen relaciones de interdependencia (Mayntz, 2006). Dado que la complejidad es una característica común de los sistemas de FP, especialmente de los sistemas duales, debido a la variedad de actores involucrados en la toma de decisiones, gestión e implementación (administraciones públicas centrales y regionales, centros formativos, empresas, agentes sociales, etc.), el estudio de su gobernanza se ha constituido en un área de investigación de interés creciente. Esto se debe a que el éxito y la eficacia de los sistemas de FP dual depende, en buena medida, del desarrollo de unas estructuras de gobernanza que favorezcan la coordinación e integración de los distintos actores que participan (Oliver, 2010).

Teniendo en cuenta estas consideraciones, este artículo pretende: 1) establecer unas dimensiones de análisis para entender y clasificar sistemas de gobernanza de FP dual; 2) comparar a través de estas dimensiones los rasgos básicos de los modelos de gobernanza de dos casos (Dinamarca y Alemania) que cuentan con sistemas de FP dual plenamente institucionalizados; 3) concretar las principales características del modelo de gobernanza del incipiente sistema español a la luz de las dimensiones de análisis propuestas y los dos casos europeos, que se utilizarán como elementos de contraste con el caso español.

Se presentan, por tanto, unas dimensiones de análisis y se compara a través de ellas la gobernanza de la FP dual en Dinamarca, Alemania y España. Se trata, no obstante, de una comparación asimétrica dado que Dinamarca y Alemania se utilizan como elementos de contraste con el caso español, el cual es analizado con mayor profundidad recurriendo, además de a un análisis normativo, a una metodología cualitativa que recurre al grupo de discusión como técnica para recabar información. Se espera, abordando estos aspectos, alentar el debate en torno a las bases sobre las cuales debería asentarse el incipiente modelo español para organizarse de la manera más eficaz y participativa posible.

La estructura del artículo es la siguiente. En primer lugar, se definen los conceptos centrales empleados en al artículo, se sintetiza el estado de la cuestión sobre la gobernanza y la FP y se establecen unas dimensiones de análisis que permiten el estudio y clasificación de distintos modelos de FP dual. En segundo lugar, se describe brevemente la metodología empleada que, en el análisis del caso español, ha recurrido, además de a un análisis normativo, a un trabajo de campo basado en grupos de discusión. En tercer lugar, se presentan los casos de Dinamarca y Alemania. En cuarto lugar, se analiza el modelo español. Finalmente, se exponen las conclusiones. 
El trabajo que aquí se expone proviene de una investigación europea financiada por la agencia europea CEDEFOP que ha contado con la participación de grupos de investigación de seis países europeos, estando liderada por la Universidad de Bremen. El estudio del caso español se llevó a cabo en el centro de estudios NOTUS².

\section{Gobernanza de la Formación Profesional: estado de la cuestión y propuesta de dimensiones de análisis}

La investigación comparada ha mostrado que la mayoría de los países europeos ha desarrollado desde hace años sistemas de FP duales que combinan de algún modo formación teórica y práctica. En contra de una percepción muy extendida, la organización «dual» de la FP no es una característica exclusiva de países como Alemania, Austria o Dinamarca. Tal atribución responde a cierta confusión terminológica presente en el discurso político de la FP.

La organización dual de la formación se basa en el argumento teórico de que las ocupaciones solo pueden aprenderse, en última instancia, en un proceso real de trabajo (Garfinkel, 1986). La aceptación de este argumento ha favorecido que la práctica totalidad de los sistemas de FP hayan institucionalizado la formación práctica en un proceso real de trabajo. En este sentido, dos modelos de FP pueden distinguirse: 1) un modelo de FP integrado o de una fase; y 2) un modelo de FP en alternancia o de dos fases (Rauner y Smith, 2010). En el primer modelo, característico de países como Alemania, Dinamarca, Austria o Suiza, la formación teórica en el centro educativo y la práctica en el centro de trabajo se alterna en cortos intervalos de tiempo. Asimismo, los estudiantes, denominados comúnmente en la terminología de estos sistemas como «aprendices», suelen tener estatus de asalariado, siendo por tanto retribuidos por su trabajo y protegidos por los derechos de la legislación laboral y la negociación colectiva. En el segundo modelo, en el que puede situarse el sistema tradicional español, una fase relativamente larga de formación impartida en los centros educativos da paso a una formación práctica, desarrollada en el centro de trabajo. En este modelo los estudiantes no disponen de contrato laboral ni remuneración si bien pueden recibir prestaciones económicas por parte del Estado, generalmente en forma de becas. Para evitar confusiones, y ciñéndonos a la terminología empleada en España, denominaremos modelo dual al primer tipo.

La gobernanza, por su parte, es un concepto que hace referencia a los elementos que permiten coordinar las actividades de diversas instituciones y actores que tienen relaciones de interdependencia. En este sentido Mayntz (2006) vincula el concepto a los mecanismos, prácticas y procesos que abordan la interdependencia de diversos actores en sistemas complejos, con vistas a coordinar sus actividades para solucionar problemas comunes o alcanzar fines compartidos. La gobernanza tendría lugar en un contexto relacional que no es, única o necesariamente, jerárquico, pudiendo derivarse de distintas formas institucionalizadas de cooperación que abarcarían desde las más horizontales, tales como la auto-regulación de organizaciones privadas, hasta aquellas que combinan diversas formas de cooperación entre actores públicos y privados y las que emanan de regulaciones impuestas por el Estado (Mayntz, 2006).

2 El autor agradece el trabajo desarrollado por el equipo del proyecto y, especialmente, la labor desempeñada por Oriol Homs, en su papel de director del estudio del caso español. 
El término gobernanza se ha usado con frecuencia en los análisis politológicos y, crecientemente, en los estudios de relaciones laborales centrados en la Unión Europea. Desde la perspectiva politológica, el enfoque de la gobernanza analiza las innovaciones político-administrativas desencadenadas con el proceso de integración europea, que se concretan en redes que incluyen estructuras de gobierno supranacional, nacional y subnacional (regional y provincial) (Hooghe y Marks, 2001; Mariscal, 2003). Los análisis de las relaciones laborales, por su parte, abordan el papel que agentes sociales transnacionales y nacionales, organizados en distintos niveles (intersectorial o sectorial) juegan, en coordinación con los actores gubernamentales europeos y nacionales, en los procesos de diseño e implementación de políticas de empleo y sociales. Desde esta perspectiva, el diálogo social europeo se representa como una constelación de relaciones horizontales y verticales entre diversos agentes, que conlleva diferentes niveles de dependencia y autonomía (Marginson y Sisson, 2004; Marginson y Keune, 2012). Tanto en los análisis politológicos como de relaciones laborales, el concepto puede remitir tanto un resultado o efecto vinculado a procesos de integración política o interdependencia, como a una variable que interfiere y condiciona dichos resultados (Marginson y Keune, 2012).

En los últimos años, el concepto de gobernanza se ha aplicado al análisis de la FP. Su aplicación en este campo suscita interés debido a que los sistemas de FP, a diferencia de los sistemas educativos, se han organizado en distintos países a través de un variado y heterogéneo grupo de actores que incluye al Estado, proveedores privados, agentes sociales, etc.; que se articulan a través de distintos niveles de gobierno (fundamentalmente nacional y regional). La variedad de organizaciones y actores involucrados responde a los distintos orígenes y objetivos de sistemas de FP integrados a su vez en contextos societales específicos. Las diversas configuraciones existentes se vinculan, a su vez, a distintos resultados, sobre todo con relación a su capacidad para favorecer la integración laboral de los estudiantes.

El análisis comparado de la gobernanza de los sistemas de FP ha puesto el foco en distintos elementos. Centrándose en los agentes que participan y las funciones que desempeñan, son comunes las tipologías de sistemas de FP que diferencian, de forma un tanto simplificada, modelos estatales, en los que prevalece la formación impartida en la escuela y ni las empresas ni los sindicatos tienen una función institucional asignada; de mercado, caracterizados por asignar el control y gestión de las cualificaciones profesionales al libre juego entre la demanda y la oferta; y corporatistas, cuyo signo distintivo es que el papel relevante que los agentes sociales desempeñan en la regulación e implementación del sistema (Clematide et al. 2005; Greinert, 2004; Homs, 2008).

Otros estudios comparados interesados en la gobernanza se han centrado en los denominados «regímenes de formación de cualificaciones» (Busemeyer y Trampusch, 2012). El término formación de cualificaciones remite a las políticas e instituciones que se ocupan de la provisión de formación y capital humano. Los regímenes de formación de cualificaciones reflejan decisiones en torno al papel que diferentes agentes como el Estado, las empresas o los trabajadores y sus representantes juegan en la provisión y financiación de la formación. Partiendo del concepto de «regímenes de formación de cualificaciones», Busemeyer y Trampusch (2012) han construido una clasificación sobre la base de dos dimensiones: el nivel de compromiso público con la formación (alto/bajo) y el grado participación de las empresas en la formación inicial (alto/bajo). De la intersección de estas dos dimensiones derivan cuatro modelos. Un modelo estatista con una baja participación de las empresas, presente en países como Francia, Suecia o España; un 
modelo colectivo que cuenta con un alto compromiso público y una elevada participación empresarial, y puede observarse en Alemania o Austria; un modelo liberal que carece de compromiso público con la formación y, si bien asigna al mercado la principal función en cuanto a la asignación y reconocimiento de las cualificaciones, el compromiso y la inversión de las empresas en formación es bajo, situado generalmente en Reino Unido; y un modelo segmentacionista en el que las empresas tienen una amplio protagonismo y dedican importantes inversiones en formación en un contexto de escasa intervención estatal, modelo que los autores identifican en el sistema japonés.

Las dimensiones de análisis que en este artículo se plantean se inspiran en la tipología de modelos de gobernanza confeccionada por Rauner y sus colaboradores en el curso de distintas investigaciones comparadas que han analizado los modelos de FP dual en distintos países (Rauner, Wittingy Deimer, 2010; Rauner y Wittig, 2010). Es decir, se trata de una tipología que, a diferencia de las anteriormente expuestas, se diseña para clasificar los sistemas de FP dual. Los modelos de FP dual se caracterizan por estar compuestos de dos sub-sistemas interrelacionados que incluyen la formación impartida en el lugar de trabajo, gestionada por empresas privadas; y el subsistema educativo de la FP, cuyas competencias recaen en el Estado. Aunque a simple vista este sistema podría parecen una combinación del modelo estatal y de mercado, lo cierto es que la mayoría de países en los que la FP dual está institucionalizada se caracterizan por poseer elementos corporatistas, otorgando importantes responsabilidades y funciones a los agentes sociales y la negociación colectiva. Reconociendo por tanto que la FP dual tiende a institucionalizarse a través de formas de gobernanza plural en las cuales pueden coincidir, al mismo tiempo, elementos de gobernanza estatal, de mercado y corporatista, estos autores crean una tipología para clasificar los sistemas de FP dual sobre la base de dos dimensiones: grado de coordinación y racionalidad de la agencia.

El grado de coordinación de los diferentes agentes remite a la integración del sistema. Un sistema de FP dual puede ser completamente fragmentado. En este caso, los agentes llevan a cabo sus funciones de manera autónoma y sin coordinación. Las responsabilidades no están distribuidas de acuerdo a funciones, lo que conlleva que las competencias de regulación, implementación y evaluación recaigan sobre varios agentes a través de diversas constelaciones. El otro extremo es un sistema plenamente coordinado que, según los autores (Rauner, Wittingy Deimer, 2010; Rauner y Wittig, 2010), no debe asociarse a un modelo estatal en el que la administración pública concentra la mayor parte de funciones y competencias en cuanto a la FP. El modelo coordinado, en el marco de un sistema de gobernanza plural característico de la FP dual, combinaría un marco legal consistente que delegaría en el Estado ciertas funciones estratégicas, tales como la regulación de las cualificaciones o estándares formativos, favoreciendo, al mismo tiempo, la participación de distintos niveles gubernamentales así como la integración de distintos actores (sindicatos, organizaciones empresariales, empresas) a los que se asignaría diversas funciones del sistema.

La segunda dimensión hace referencia a la racionalidad de la agencia, entendida como el conjunto de principios que guían el comportamiento de los distintos agentes. En este sentido, se establece una diferencia entre una racionalidad basada en normas o «inputs» y una racionalidad orientada hacia los resultados o outcomes. La racionalidad basada en normas se enraíza en el modelo tradicional burocrático, centrado en la adecuación de las acciones a los criterios legales. La racionalidad basada en resultados, por el contrario, se vincula a la aproximación de la Nueva Gestión Pública (Osborne y Gaebler, 1993; Spicer 
2004) que, a grandes rasgos, propone exportar las técnicas de gestión privada a la administración públicas en aras de mejorar la eficiencia.

Las dos dimensiones de análisis sugeridas permiten construir una matriz (ver tabla 1) cuyos cuatro cuadrantes representan diferentes modelos de gobernanza (fragmentado basado en las normas, coordinado basado en las normas, fragmentado orientado hacia resultados).

Tabla 1. Tipología de modelos de gobernanza. Fuente: Rauner, Wittingy y Deimer, 2010; Rauner y Wittig, 2010.

\begin{tabular}{|c|c|c|c|}
\hline \multicolumn{4}{|c|}{ Grado de coordinación (integración) } \\
\hline \multirow{3}{*}{ 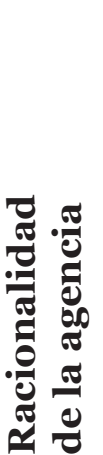 } & & Bajo & Alto \\
\hline & 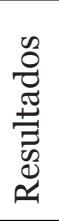 & $\begin{array}{l}\text { Fragmentado } \\
\text { orientado hacia } \\
\text { resultados }\end{array}$ & $\begin{array}{l}\text { Coordinado orientado } \\
\text { hacia resultados }\end{array}$ \\
\hline & 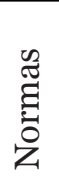 & $\begin{array}{l}\text { Fragmentado basado } \\
\text { en las normas }\end{array}$ & $\begin{array}{l}\text { Coordinado basado } \\
\text { en las normas. }\end{array}$ \\
\hline
\end{tabular}

Sobre la base de esta tipología, se han concretado 4 dimensiones de análisis que permiten una distinción un tanto más precisa y diversificada de los sistemas de gobernanza que la matriz presentada en la tabla 1, estructurada en torno a las dos dimensiones y que, en la práctica, supone mantener la dimensión relativa a la racionalidad de la agencia; dividir la dimensión concerniente al grado de coordinación en dos nuevas dimensiones; y añadir una dimensión referente al grado de participación de los agentes sociales. La inclusión de esta última dimensión se estima necesaria para poder trasladar el análisis a países en los cuales el diálogo social referente a la formación está menos institucionalizado y, en consecuencia, puede verse alterado en función de la coyuntura política y económica, como ocurre en cierta medida en el caso de España. País el cual, además, el carácter incipiente del modelo puede implicar que los agentes sociales no tengan asignadas unas funciones específicas o estas estén en proceso de definición. Las cuatro dimensiones que se emplean en el análisis son:

- Consistencia del marco legal. Esta dimensión analiza la distribución de las competencias legislativas y la complementariedad e integración de las distintas regulaciones que pueden existir en los diferentes niveles administrativos (estatal, regional o local) y subsistemas formativos (formación en el aula y formación en la empresa). Asumiendo que tanto un modelo centralizado como descentralizado puede dotarse de un marco legal consistente, el foco de atención recae sobre el grado de coordinación o fragmentación del sistema.

- Participación / Integración de los agentes sociales. Esta dimensión analiza en qué medida el sistema permite la participación de los agentes sociales. Se diferencia así un modelo integrador que permite la participación de sindicatos 
y organizaciones empresariales o empresas en los distintos niveles (estatal, regional y local) de un modelo excluyente que niega la participación a los agentes sociales o la restringe a determinados actores o niveles.

- Asignación de funciones estratégicas y operativas. Esta dimensión se interesa por el tipo de asignación de las responsabilidades relativas al desarrollo de las cualificaciones y el diseño de los currículos entre los distintos agentes (administraciones centrales, regionales y locales y centros formativos). Se distingue, en este sentido, una asignación de funciones centralizada en la cual todas las funciones, tanto las estratégicas como las operativas, recaen sobre un órgano estatal, de una asignación articulada sobre el principio de subsidiariedad, en la cual las decisiones estratégicas se concentran a nivel estatal mientras que las operativas se adoptan lo más cerca posible del entorno local.

- Racionalidad de la agencia. Esta dimensión analiza la orientación del sistema, distinguiendo una racionalidad basada en normas o «inputs» y una racionalidad orientada hacia los resultados o «outcomes».

\section{Metodología}

En función de las cuatro dimensiones anteriormente expuestas se analizan en los apartados posteriores los casos de Dinamarca, Alemania y España. Se trata, como se ha apuntado en la introducción, de una comparación asimétrica, dado que Dinamarca y Alemania se toman como elementos de contraste con el caso español, que se analiza con mayor profundidad. Atendiendo a este fin, se han empleado también distintas técnicas de recogida de información, dentro de una perspectiva de investigación cualitativa.

La exposición de los casos de Dinamarca y Alemania se basa únicamente en un análisis normativo resultante de una revisión bibliográfica. En dicha revisión se han consultado los trabajos de Rauner et al. (2010) y Rauner y Wittig (2010), en los cuales aplican su tipología de sistemas de gobernanza de FP dual explicada en el apartado anterior a los casos de Dinamarca y Alemania. Se trata por tanto de trabajos fundamentales para los objetivos de este artículo habida cuenta de que las dimensiones de análisis propuestas se inspiran, principalmente, en las contribuciones de estos autores. Esta información se ha completado con trabajos procedentes de otros autores. Por una parte, se han consultado los informes nacionales de Dinamarca y Alemania elaborados por la red de expertos de CEDEFOP «REFERNET», los cuales contienen información precisa sobre las características institucionales de cada modelo, resultando especialmente pertinentes para obtener la información descriptiva sobre la que sustentar y basar el análisis de sus estructuras de gobernanza. Estos informes se encuentran publicados en la web de la citada agencia europea. Por otra parte, se ha desarrollado una búsqueda de bibliografía relevante mediante la introducción de diferentes combinaciones de palabras clave (governance + dual VET + Denmark; governance + dual VET + Germany; etc.) en el portal de la biblioteca digital de una universidad pública que facilita el acceso a los recursos electrónicos (libros y revistas) de relevantes bases de datos académicas (Emerald Journals, Sage, Dialnet, SciELO, Scopus, etc.). Esta búsqueda produjo un mayor número de resultados para el caso alemán, pero permitió detectar al menos dos artículos particularmente relevantes para el análisis de Dinamarca que, pese a no centrarse 
primordialmente en la gobernanza, contienen observaciones trascendentes sobre la evolución del papel y funciones de los diferentes agentes que participan en el sistema. Cabe destacar que la búsqueda de literatura permitió constatar que la gobernanza no ha sido el principal objetivo de investigación de la FP dual en estos países, predominando en los últimos años publicaciones centradas en el análisis y la evaluación de sus resultados.

$\mathrm{El}$ análisis de las estructuras de gobernanza del recientemente regulado sistema de FP dual español se ha fundamentado, por un lado, en un análisis normativo, basado en una revisión de los documentos técnicos y legislativos más relevantes. Este estudio normativo se ha combinado con un análisis de las opiniones y percepciones de los distintos actores. La escasa institucionalización del sistema de FP dual español requería, según nuestro criterio, acercarnos a los principales actores que están participando en su desarrollo para clasificarlo adecuadamente a la luz de las dimensiones propuestas. Se recure así a una perspectiva de investigación cualitativa que aspira a comprender el fenómeno a partir de datos que proporcionan los individuos que participan en el diseño e implementación del sistema, considerando sus puntos de vista como relevantes (Mena y Menéndez, 2009).

Para recopilar la información relativa a la percepción de los distintos actores se ha recurrido a la técnica de recogida de información de los grupos de discusión. La elección de esta técnica reside en la importancia otorgada en la investigación al estudio de los discursos en un ambiente grupal, que favorece el intercambio equilibrado de opiniones entre los distintos actores, permitiéndoles enfrentar y contrastar sus valoraciones de forma que el discurso de unos pueda provocar reacciones y dar lugar a aclaraciones (Mena y Menéndez, 2009). En este sentido, conviene destacar la pluralidad de actores involucrados en la FP dual (administraciones públicas, empresas, sindicatos, etc.). Teniendo en cuenta sus distintas posiciones e intereses, se asume que pueden tener ideas diferentes y enfrentadas sobre las estructuras óptimas de gobernanza. El grupo de discusión permite así enfrentar y contrastar sus visiones, con vistas a identificar y analizar los puntos de vista de los diversos actores. Por otro lado, cabe destacar que esta técnica puede favorecer, a través de la discusión, la modificación de puntos de vista o el reconocimiento de ideas (Mena y Menéndez, 2009). Un aspecto especialmente relevante a la hora de abordar un modelo en proceso de institucionalización cuyas características intrínsecas requieren, para su correcto desarrollo y funcionamiento, de cierto consenso con relación a sus objetivos y la distribución de funciones y competencias. El grupo de discusión permite así analizar de manera más precisa que mediante otras técnicas como las entrevistas no solo los posibles acuerdos y disensos en torno la gobernanza de la FP dual, sino también las posibilidades de alcanzar compromisos que puedan satisfacer a las distintas partes.

Los grupos de discusión se implementaron del siguiente modo. Se realizaron 3 grupos de discusión en dos sesiones concretadas en junio y octubre de 2014. Cada sesión contó con la participación de 36 personas (con 12 personas en cada grupo) provenientes de la administración pública (Ministerio de Educación y Consejerías de Educación y Empleo); los centros de formación profesional (directores de centro y profesores); agentes sociales de ámbito estatal y autonómico; y empresarios involucrados en los programas de formación dual. Cada grupo de discusión se confeccionó buscando una presencia homogénea de los 4 colectivos mencionados. Con este diseño se buscaba, como se ha apuntado, favorecer el intercambio equilibrado de opiniones entre los distintos actores, permitiéndoles enfrentar y contrastar sus valoraciones. Complementariamente, se perseguía analizar, a través de este diálogo, el discurso de cada colectivo con relación a la gobernanza del nuevo sistema de FP dual en función de sus distintas posiciones e 
intereses, así como los principales elementos de consenso y disenso. La discusión de los grupos se estructuró de acuerdo a las cuatro dimensiones de análisis propuestas, las cuales se operativizaron en una serie de cuestiones abiertas destinadas a animar y orientar el diálogo.

En pos de la brevedad, se ha optado por presentar los resultados del trabajo de campo de manera comprehensiva, evitando reproducir citas de los grupos de discusión. A pesar de esto, se distinguen las opiniones expresadas por los distintos actores.

\section{Gobernanza y Formación Profesional dual en Europa: los casos de Dinamarca y Alemania}

El caso de Dinamarca representa una forma clásica de FP dual poco extendida en el contexto nórdico, al estar separada de la vía educativa general. Dentro de este sistema, el aprendizaje en el centro de trabajo representa dos tercios del total que los alumnos reciben a lo largo de un ciclo académico de cuatro años. El sistema proporciona una efectiva transición al mercado de trabaja, contribuyendo a situar el desempleo juvenil en niveles sustancialmente inferiores a los de la mayoría de países europeos (Helms, 2013).

En términos de gobernanza, el caso danés puede ser visto como un ejemplo de un modelo coordinado y participativo, que asigna las funciones de acuerdo con el principio de subsidiariedad (Raune y Wittig, 2010). Su marco legal concentra la responsabilidad política en una sola institución, el Ministerio de Educación, que supervisa los centros formativos y ostenta las competencias sobre los denominados cursos básicos de FP. Además, existe una única ley que regula todo el sistema (Vocational Education and Training Act) y se complementa con regulaciones que abordan elementos específicos tales como la autorización y el funcionamiento del sistema formativo.

La concentración de las principales responsabilidades políticas en una única institución estatal no significa, empero, que todas las funciones del sistema estén centralizadas. Por el contrario, estas se asignan de acuerdo con el principio de subsidiariedad. Las funciones estratégicas del sistema, como la concreción de los perfiles ocupacionales y las cualificaciones profesionales, se concentran a nivel estatal. En este ámbito, los agentes sociales (federaciones sindicales y patronales sectoriales) desempeñan un rol central, creando y renovando las cualificaciones profesionales de manera ágil, a través de comités nacionales sectoriales. A nivel local se delegan las funciones operativas, que incluyen el desarrollo de los currículos formativos y los planes formativos individuales. Los principales actores en este nivel son los centros formativos (vocational colleges), las empresas y los comités de formación profesional locales, que incluyen también representantes de los agentes sociales. De este modo, el modelo permite un alto grado de participación de los agentes sociales (Rolls, 2012). El alto nivel participación de los agentes sociales en la gobernanza, otorga legitimidad y reconocimiento social al sistema, en un contexto en el cual la ocupación, definida a través de la cualificación, es el principio fundamental que estructura la demarcación de los sindicatos (Helms, 2013)

En cuanto a la racionalidad de la agencia, el modelo danés se caracteriza por una orientación basada en los resultados, concretada a través de diferentes reformas implementadas desde la década de los 90 (Juul y Helms, 2011). Existe en este modelo un programa nacional de objetivos e indicadores que orienta a los actores locales y permite la evaluación de sus acciones. El sistema otorga, además, autonomía a los centros formativos 
para adaptar perfiles ocupacionales generales que son definidos a escala nacional. En este modelo, los centros formativos son instituciones independientes que gestionan sus presupuestos y concretan los planes curriculares y los planes individuales formativos, adaptándolos al contexto local (Raune y Wittig, 2010). Esta orientación y, especialmente, el creciente énfasis en la individualización de los planes formativos, puede, tal y como alertan críticamente Wiborg y Cort (2010), contribuir a que se limite progresivamente el papel de los agentes sociales en el sistema, derivando así en un modelo más excluyente. No obstante, otros autores (Juul y Helms, 2011; Helms, 2013) constatan que la mayor autonomía otorgada a los centros formativos a lo largo de los últimos años no ha limitado la participación e importancia de los agentes sociales. Así, las competencias otorgadas a los centros formativos para ajustar los programas al entorno local, permitiéndolos incluso ofrecer planes formativos flexibles y alternativos a los definidos en los comités sectoriales nacionales, no han tenido apenas impacto, precisamente, por la falta de reconocimiento de los agentes sociales.

Alemania es probablemente el modelo más estudiado y conocido de FP dual, atribuyéndoosle generalmente un papel fundamental en el fortalecimiento de la competitividad económica de este país, así como en el mantenimiento de tasas de desempleo juvenil comparativamente bajas (Jacob y Solga, 2015). En este sistema, la formación dura entre dos y tres años, ocupando el aprendizaje en la empresa tres o cuatros días a la semana (Solga, Protsch, Ebner y Christian Brzinsky-Fay, 2014).

En lo concerniente a la gobernanza, el modelo alemán representa el ejemplo de un marco jurídico relativamente fragmentado (Rauner y Wittig, 2010), como pone de manifiesto la separación de las competencias reguladoras y de gestión de la formación teórica y la formación en la empresa en distintas instituciones emplazadas, a su vez, en diferentes niveles territoriales. Así, las competencias relativas a la formación teórica impartida en las escuelas y centros formativos recaen en los gobiernos regionales (Länder). Las competencias de la ordenación de la formación en la empresa (objetivos, contenidos, criterios de evaluación, etc.) están, por el contrario, bajo el dominio del gobierno federal. Así, la regulación de la formación en la empresa para las ocupaciones reconocidas es desarrollada por el Ministerio de Economía y Tecnología (BMWi), actuando en colaboración con el Ministerio de Educación e Investigación (BMBF). El registro de los contratos formativos, las tareas de supervisión y aprobación de las empresas capacitadas para impartir formación dual, y las competencias relativas a la evaluación de dicha formación, recaen en los denominados «competent bodies», que incluyen principalmente a las cámaras de comercio, que deben integrar a los representantes sindicales en fases tales como la evaluación de los contenidos formativos (BIBB, 2011; Hippach Schneider y Huismann, 2016). El capital papel de las cámaras de comercio en la implementación de la formación en la empresa, que se remonta a finales del siglo XIX, constituye una de las principales señas de identidad del sistema alemán (Deissinger y Gonon, 2016). Encontramos, pues, a un heterogéneo grupo de departamentos gubernamentales emplazados a nivel estatal y regional que actúa en coordinación con actores no gubernamentales (principalmente cámaras de comercio y empresas). Esta distribución genera, en la práctica, problemas en cuanto a la coordinación de los centros formativos y las empresas. Así, una encuesta realizada a 1.600 «aprendices» mostró que dos tercios de estos calificaron la cooperación entre empresas y centros educativos como «poco o nada efectiva» (Rauner y Wittig, 2010). 
En cuanto al grado de participación, se trata de un sistema integrador en el cual los agentes sociales intervienen tanto en la definición de las ocupaciones y cualificaciones profesionales a escala estatal, como en la regulación de las condiciones laborales de los aprendices, mediante la negociación colectiva sectorial (Solga et al. 2014). Es destacable, asimismo, el papel de los sindicatos en la planificación e implementación de la formación. También participan en la evaluación de los aprendices, estando representados en los comités examinadores gestionados por las cámaras de comercio (Hippach Schneider y Huismann, 2016). El histórico papel del sindicalismo en el sistema es resaltado por Deissinger y Gonon (2016), quienes le atribuyen una función esencial en su conservación. Como destacan estos autores, en el modelo alemán, los sindicatos y sus distintas federaciones sectoriales tienen desde hace décadas una participación institucionalizada con relación a distintas funciones estratégicas. Esta integración les ha permitido consolidar un conjunto de creencias y valores políticos en torno al valor y la función social de la FP dual que dota de legitimidad al sistema y ha asegurado su pervivencia a lo largo de los años.

Respecto a la asignación de funciones, puede decirse que el sistema alemán, a pesar de su fragmentación, tiende a concentrar sus funciones operativas y estratégicas a nivel estatal (Raune y Wittig, 2010; Rauner et al. 2010). Así, la regulación y aprobación de las denominadas ocupaciones reconocidas se lleva a cabo a nivel estatal a través de ordenanzas aprobadas por el BMWi, en cooperación con el BMBF y previo consenso con los agentes sociales. Conviene destacar, en este sentido, que ninguna de las ocupaciones reconocidas puede ser regulada sin la aquiescencia de los agentes sociales quienes, además, ostentan legalmente la iniciativa para proponer el desarrollo o actualización de las mismas. Esta regulación incluye aspectos tales como la definición de la ocupación, la duración de la formación que da acceso a la titulación, las cualificaciones y capacidades requeridas para su desempeño, el plan formativo y los criterios de evaluación (Hippach Schneider y Huismann, 2016). De este modo, los centros formativos y órganos locales con competencias en la evaluación de la formación («competent bodies») tienen poco margen de autonomía, si bien esta ha aumentado desde 2005, permitiendo a las empresas que proveen formación ampliar el programa con «cualificaciones adicionales» que son evaluadas y reconocidas (BIBB, 2011).

La rigidez con que se definen los perfiles ocupacionales y sus necesidades formativas a escala estatal, dando lugar a un modelo curricular excesivamente especializado en el que existen más de 300 ocupaciones reconocidas, lleva a Raune y Wittig (2010) y Rauner et al. (2010) a calificar la racionalidad de la agencia del sistema alemán como burocrática u orientada hacia el cumplimiento de las normas, en contraste con el caso danés.

\section{Gobernanza y Formación Profesional dual en España}

\subsection{Consistencia del marco legal}

Un primer aspecto a aclarar es que el gobierno optó por regular el nuevo modelo de formación profesional dual a través de un Real Decreto, norma jurídica cuya aprobación corresponde al ejecutivo. En este sentido, es reseñable que el Real Decreto 1529/2012, de 8 de noviembre, por el que se desarrolla el contrato para la formación y el aprendizaje y 
se establecen las bases de la formación profesional dual fue redactado conjuntamente por el Ministerio de Educación y Empleo. Dado que la FP dual abarca, según dicho RD, tanto la actividad formativa inherente a los contratos para la formación y aprendizaje, cuya formalización y seguimiento corresponde al Servicio Público de Empleo, dependiente a su vez del Ministerio de Empleo, como la FP desarrollada en el ámbito del sistema educativo, cuyas competencias recaen en el Ministerio de Educación, nos encontramos con dos órganos administrativos a escala estatal que ostentan competencias legislativas y de seguimiento. Tal cooperación permitiría avanzar en la consecución del objetivo, reiterativo en el tiempo, de integrar la FP inicial, dependiente del Ministerio de Educación, y la formación para el empleo, dependiente del Ministerio de Empleo. No obstante, cabe destacar que, en la práctica, la participación de los dos Ministerios ha consagrado un modelo que diferencia dos perfiles, el del estudiante de la formación inicial, dependiente de Educación, y el del «aprendiz» que puede ser contratado con el contrato de formación y aprendizaje, sujeto a la legislación laboral, dependiente de Empleo.

En los grupos de discusión se evidenció que la diferenciación entre los dos perfiles (estudiante/aprendiz), se entronca en una concepción específica y no coincidente del estatus de la FP entre las administraciones educativas y de empleo. Así, se constató que desde el Ministerio de Educación y algunas consejerías autonómicas de este ámbito existen reticencias a la hora de considerar el proceso formativo en la empresa como un empleo, circunstancia que explica la contemplación de distintas vías que no otorgan a los alumnos las garantías y derechos que ofrece la regulación laboral. En este ámbito se observan también, como cabría esperar, discrepancias entre los agentes sociales. Así, los sindicatos se muestran proclives a una regulación que solo reconozca el estatus de aprendiz, garante de derechos laborales y sociales (desempleo y pensión). Por el contrario, las organizaciones empresariales, si bien aceptan la existencia de una vía laboral, enfatizan los costes en los que incurren las empresas en el proceso formativo y reclaman mantener ambas vías y conceder flexibilidad a los empleadores en función de sus necesidades y recursos.

En congruencia con el modelo descentralizado de administración del sistema educativo español, las competencias de gestión y administración recaen en los departamentos de educación de las Comunidades Autónomas (CCAA). De manera conjunta, intervienen también las consejerías de empleo y los Servicios Públicos de Empleo Autonómicos, los cuales deben autorizar la actividad formativa relativa al contrato de formación y aprendizaje. Encontramos pues que la distribución de competencias entre dos Ministerios se reproduce a nivel autonómico, dificultando la coordinación e integración del sistema. De manera similar al modelo alemán, el sistema español otorga competencias a distintos Ministerios y Consejerías emplazados en distintos niveles territoriales sin crear mecanismos para su coordinación.

Respecto a la unidad y consistencia del marco jurídico, cabe destacar que el Real Decreto 1529/2012 por el que se desarrolla el contrato para la formación y aprendizaje y se establecen las bases de la FP dual coexiste con diferentes normas autonómicas que están actualmente en vigor. Por añadidura, algunas CCAA como Madrid tienen en funcionamiento un programa de FP dual que no ha sido regulado mediante ninguna normativa o reglamentación. Esta situación encaja con la normativa española según la cual las CCAA ostentan competencias normativas de desarrollo de las normas estatales y de regulación de los elementos o aspectos no básicos del sistema educativo. En este contexto de descentralización administrativa, la coexistencia de normas estatales y autonómicas no tiene porqué generar inconsistencia. 
No obstante, encontramos en este caso que el RD 1529/2012 apenas concreta cuestiones clave dentro de un sistema de FP dual como la relación entre el aprendiz/ estudiante y la empresa, contemplando posibilidades que van desde el contrato de formación y aprendizaje, ampliamente detallado en el $\mathrm{RD}$, hasta las becas. Como consecuencia, encontramos, por ejemplo, que en el País Vasco (Resolución 2014/691), la relación entre el aprendiz y la empresa se regulaba hasta 2015 únicamente mediante el contrato de formación y aprendizaje ${ }^{3}$ mientras que, en Madrid, los aprendices reciben una beca de $300 €$ y, en el caso de Cataluña (ENS/1204/2012), esta puede regularse a través de un contrato laboral ordinario, un contrato de formación y aprendizaje, una beca o una relación de voluntariado. En los grupos de discusión, representantes sindicales y de las administraciones educativas autonómicas subrayaron el agravio comparativo existente entre un estudiante en régimen de «voluntariado» y un «aprendiz» contratado mediante el contrato de formación. Elementos que podrían cuestionar el principio de igualdad de derechos.

Las discusiones revelaron también que otros aspectos centrales del modelo de formación dual como el tiempo de la alternancia entre el centro educativo y la empresa o los niveles incluidos en la formación dual varían también entre diferentes CCAA.

El RD tampoco regula los mecanismos de cooperación entre los centros de FP (Institutos de Enseñanza Secundaria, etc.) y las empresas. Estos mecanismos se están regulando a nivel autonómico, dando lugar, también en este ámbito, a diversas estrategias, como revelan los ejemplos de Cataluña, País Vasco y Madrid que salieron a colación en las discusiones. En el caso de Cataluña, las empresas deben suscribir con los centros educativos convenios de colaboración que deben presentarse al Departament d'Ensenyament. Según la información provista por el representante del Departament d'Ensenyament, las empresas interesadas deben acudir directamente a los centros que impartan formación circunscrita a su sector y acordar las modalidades de la colaboración que, como hemos apuntado, comprenden una elevada gama de posibilidades. En el País Vasco no existe esta figura. En esta CCAA la dirección de los centros de formación debe presentar los proyectos a la Dirección de Formación y Aprendizaje del Departamento de Educación. En Madrid, por el contrario, existe un número limitado de centros autorizados para impartir la formación dual. Las empresas que deseen participar en proyectos de formación dual deben inscribirse en una plataforma. De este modo, la conexión entre los centros y empresas es gestionada por la Consejería de Educación de la Comunidad de Madrid. Por lo tanto, se observa que la institucionalización de la cooperación entre la empresa y el centro formativo está siguiendo distintas vías.

En consecuencia, existía un amplio consenso entre los participantes en torno a la idea de que el marco legal actual está ampliamente fragmentado y carece de consistencia, permitiendo, tal y como se constató, la emergencia de distintas propuestas en las CCAA que difieren entre sí en aspectos claves y ofrecen distintas oportunidades a los aprendices y las empresas. Se trata por tanto de un sistema experimental que otorga libertad a las CCAA para adaptarlo a su realidad y está permitiendo en la práctica la existencia de distintos modelos.

3 Esta normativa fue revisada en 2015 mediante el DECRETO 83/2015, de 2 de junio, por el que se establece la Formación Profesional Dual en Régimen de Alternancia en la Comunidad Autónoma del País Vasco. Este decreto contempla una vía no laboral en la cual los aprendices reciben, en lugar de un salario, una beca para programas formativos. 


\subsection{Grado de participación}

En lo que concierne a la participación de los agentes sociales, podemos constatar, en primer lugar, que la FP dual se ha desarrollado y se está desarrollando en ausencia de un diálogo con los agentes sociales, a pesar de que, como subrayaron sus representantes en los grupos de discusión, existe un importante bagaje de diálogo social bipartito y tripartito en materia de formación en España, plasmado en distintos acuerdos formalizados desde los 80.

La ausencia de implicación de los agentes sociales en el diseño de la FP dual está generando, junto con otros elementos de su regulación, un disenso entre sindicatos y patronal. Así, en los grupos de discusión se constató que el modelo de FP dual genera expectativas positivas entre las organizaciones empresariales, siendo contemplado con escepticismo e incluso rechazo por los representantes sindicales. Las aportaciones de los representantes sindicales a lo largo de los grupos de discusión denotan la existencia de un discurso sindical que sitúa el desarrollo de la FP dual dentro de «las políticas desreguladoras y precarizadoras» que el gobierno del PP habría puesto en marcha para favorecer la inserción de los jóvenes. La crítica sindical del modelo contrasta con el alto nivel de aceptación sindical de que goza en Alemania y Dinamarca. Una aceptación que se vincula, entre otros aspectos, al papel relevante que sus respectivos modelos les otorgan.

En cuanto al papel de los agentes sociales en la definición de las cualificaciones profesionales, los grupos de discusión revelaron que existe un consenso amplio en torno a la ida de que su participación está bastante institucionalizada. Los agentes sociales contribuyen, a través de su participación en el Consejo General de la Formación Profesional, al desarrollo del Catálogo Nacional de Cualificaciones, nombrando a los expertos que participan en los 26 grupos profesionales que definen y renuevan las cualificaciones.

Finalmente, las discusiones mostraron que los diversos proyectos de FP dual están consagrando la participación de distintas constelaciones de actores a nivel local. Así, en algunas provincias y comarcas, los centros educativos están colaborando con organizaciones empresariales regionales o provinciales. En otros casos, la colaboración se da entre los centros de formación y las empresas a nivel individual, sin la mediación de organizaciones empresariales. También se mencionaron ejemplos en los cuales la administración local colabora en la concreción de proyectos de FP dual con centros y empresas. En cuanto a los sindicatos o los órganos de representación unitaria de los trabajadores en la empresa, su participación a este nivel parece carecer de relevancia. Aspecto este que la regulación actual no ayudaría a revertir, al no concederles ninguna función. La escasa implantación de los sindicatos a este nivel contrasta también con los casos de Alemania y Dinamarca.

\section{5•3. Asignación de funciones operativas y estratégicas}

La asignación de algunas de las principales funciones operativas y estratégicas dentro de un sistema de FP tales como la detección, descripción y organización de las cualificaciones profesionales o el diseño de los currículos de los títulos de formación, es una cuestión que no ha sido modificada por la normativa que regula la formación dual. La asignación de estas funciones está regulada por diferentes normativas tales como la Ley 5/2002 de las Cualificaciones y la Formación Profesional, el Real Decreto 1538/2006, de 15 de diciembre, por el que se establece la ordenación general de la formación profesional del sistema educativo o el Real Decreto 1147/2011, de 29 de julio, por el que se establece la 
ordenación general de la formación profesional del sistema educativo. En este sentido, se asume que la FP dual debe desarrollarse sobre la base de la distribución actual de competencias entre los diferentes organismos y niveles territoriales. Veamos brevemente sus características y la valoración de los distintos agentes obtenida en el trabajo de campo.

Las competencias en cuanto a la definición de las cualificaciones profesionales se concentran a nivel nacional. El Catálogo Nacional de Cualificaciones Profesionales constituye la base para diseñar la oferta formativa de los títulos de FP (establecidos por el Ministerio de Educación). Este instrumento tiene por objetivo detectar las cualificaciones profesionales más importantes del sistema productivo español y describirlas atendiendo a las competencias necesarias para el ejercicio profesional, a fin de organizarlas en familias profesionales y niveles, siguiendo de este modo una perspectiva fundamentalmente de demanda. El Instituto Nacional de las Cualificaciones (INCUAL) es el órgano encargado de la coordinación del Catálogo Nacional de Cualificaciones. Al mismo tiempo, el Consejo General de la Formación Profesional (CGFP), que representa a las distintas administraciones y los agentes sociales, ostenta funciones consultivas. Las cualificaciones profesionales se diseñan por expertos en el campo de la formación profesional a través de 26 grupos profesionales que corresponden a cada una de las familias profesionales reconocidas.

La concentración de las competencias relativas al diseño de las cualificaciones en estos organismos es un aspecto que, a grandes rasgos, goza de consenso. Así, de las discusiones se desprende la asunción, compartida por los diversos actores, de que dichas competencias deben concentrarse a nivel estatal para garantizar el valor y uniformidad de las cualificaciones en todo el Estado. También se estima, en líneas generales, que la organización del diseño del Catálogo a través de los grupos de trabajo garantiza la participación de expertos profesionales en la definición de las cualificaciones. La principal crítica con relación a este organismo, formulada por la mayor parte de los participantes, remite a la rigidez del Catálogo, y su falta de dinamismo para actualizar y renovar las cualificaciones. Su falta de imbricación en la negociación colectiva fue un problema resaltado también por los agentes sociales (tanto sindicatos como organizaciones empresariales).

En cuanto la elaboración del currículo de los títulos de FP, los aspectos básicos son regulados por el Ministerio de Educación, sobre la base del Catálogo Nacional de Cualificaciones, reservándose la ampliación y desarrollo de estos contenidos a las CCAA. La regulación no otorga a los centros que pueden impartir FP competencia alguna en la elaboración de los currículos de los ciclos formativos, limitando su papel a su aplicación. A pesar de esto, la tendencia de las últimas regulaciones, como Real Decreto 1147/2011, se orienta a promover cierta autonomía pedagógica, organizativa y de gestión a los centros. Por otro lado, se ha de destacar que los centros que imparten FP han tenido autonomía a la hora de concretar el módulo profesional de formación en centros de trabajo (FCT).

Asumiendo estas restricciones, que denotaría un tipo de asignación de funciones estratégicas y operativas de carácter centralizado, profesores y directores de centros que imparten FP resaltaron que disponen de un alto grado de autonomía para adaptar la nueva formación dual al entorno productivo local. Así, las discusiones permitieron conocer distintos ejemplos de proyectos de FP dual promovidos por los propios centros de formación. En estos casos, los centros han aprovechado relaciones consolidadas a través de los módulos de FCT u otras experiencias autonómicas menos conocidas como las prácticas de profesores de FP en las empresas para concretar los nuevos proyectos. Por ejemplo, en una comarca catalana, el Instituto de Educación Secundaria que imparte los 
ciclos de formación de productos agroalimentarios ha formalizado un acuerdo con seis empresas cárnicas de la zona con las que venía colaborando en los módulos de FCT desde hacía años para impartir la formación dual integrada desde el año 2013. El amplio conocimiento que los profesores disponen del proceso de trabajo y requerimientos formativos de estas empresas, tras años de colaboración, habría permitido transitar de forma fluida al régimen dual. En otras regiones, los centros encuentran más obstáculos. Algunos de los problemas mencionados remiten al tamaño de las empresas españolas. En este sentido, tanto profesores como representantes de organizaciones empresariales destacaron que las empresas pequeñas carecen en muchos casos de la información y los recursos necesarios para participar con garantías en un proyecto de FP. Frente a estos obstáculos, profesores y centros de FP demandan un mayor apoyo institucional y de otros agentes (organizaciones empresariales, cámaras de comercio, etc.).

Al hilo de las discusiones sobre la autonomía de los centros aparecieron también ejemplos en los que las empresas habían constituido centros de FP privados reconocidos por la Administración. Así, se comprobó que empresas multinacionales del sector automovilístico han creado sus propios centros de formación para impartir distintos ciclos formativos oficiales (mecanizado, mantenimiento electromecánico, etc.) en régimen dual, de acuerdo con la nueva normativa. Si bien se valoró positivamente su contribución a la promoción de la inserción laboral de los jóvenes, dado que uno de los objetivos de esta formación es facilitar las tareas de selección y reclutamiento de trabajadores, distintas dudas y críticas fueron formuladas por representantes de los sindicatos, de los centros de formación públicos y algunas administraciones autonómicas. Así, se criticó la posibilidad de que estos centros estuvieran realizando una adaptación curricular a sus propias necesidades formativas al margen de los currículos establecidos por el Estado y las CCAA, beneficiándose al mismo tiempo de recursos públicos. Por otro lado, el hecho de que en algunos de estos ciclos formativos solo se garantiza un salario mensual de 250 euros el primer año y 420 el segundo, es decir, inferior al Salario Mínimo Interprofesional, mereció críticas sindicales a las condiciones laborales en las que se estaba basando la inserción de los estudiantes, reprochando en este sentido a la administración que la FP dual estaba permitiendo a estas empresas ahorrar recursos en formación y disponer de una mano de obra barata.

Dejando de lado estos ejemplos controvertidos, la mayor parte de los participantes en los grupos de discusión se mostró satisfecha con la actual distribución de competencias y funciones. Una distribución que se considera adaptada al principio de subsidiariedad y otorga, en la práctica, un margen considerable de autonomía a los centros para formalizar proyectos duales adaptados al entorno productivo. Un entorno productivo que los profesores y directores de centros de FP afirman conocer tras años de experiencia en la búsqueda de empresas con las que formalizar prácticas para los alumnos o incluso estancias de formación para los propios profesores.

\subsection{Racionalidad de la agencia}

Del análisis de la racionalidad de la agencia, las siguientes consideraciones pueden formularse. Por un lado, parece evidente que el sistema de FP dual español no está orientado formalmente hacia la consecución de resultados. Así, no existe por el momento, como ocurre en países que ejemplifican esta orientación como Dinamarca, un programa nacional de objetivos e indicadores que oriente a los actores locales y permita la evaluación de sus acciones. Tampoco está prevista una evaluación sistemática de los logros u objetivos 
alcanzados por las distintas CCAA y, dada la reciente implantación del sistema, es imposible juzgar si los resultados de la investigación o de las evaluaciones realizadas por agentes externos a la administración se tienen en cuenta en la toma de decisiones políticas.

Sin embargo, la mayoría de los asistentes a los grupos de discusión manifestó que es difícilmente asumible que este modelo tenga una orientación burocrática. Como se ha puesto de manifiesto anteriormente, la falta de concreción de la normativa actual permite un amplio margen de maniobra a las instituciones autonómicas y actores locales (empresas y centros de formación) para experimentar con distintas aproximaciones y fórmulas a través de las cuales los alumnos puedan alternar la formación en la empresa y en el centro de trabajo. La nada restrictiva definición de formación dual recogida en el RD 1529/2012 refuerza aún más esta idea. Habida cuenta de esta realidad, el sistema actual español representaría un caso atípico que, careciendo de una regulación rígida que constriñe a los actores autonómicos y locales, tampoco dispone de una estrategia clara que los oriente para conseguir la consecución de resultados deseables. De este modo, más que situarse en una posición intermedia entre una orientación basada en normas y una basada en resultados, observamos que, en el caso español, la racionalidad de la agencia no está definida.

\section{Conclusiones}

De lo anteriormente expuesto se concluye que el modelo de gobernanza del incipiente sistema español de FP dual puede definirse como altamente fragmentado, puesto que posee un marco legal inconsistente que en la práctica permite la coexistencia de distintos sistemas autonómicos y se desarrolla a través de un heterogéneo grupo de instituciones y actores que ostentan competencias. Es por tanto un modelo que guarda algunas similitudes con el caso alemán en este aspecto (Rauner y Wittig, 2010).).

En cuanto al nivel de integración de los agentes sociales, el sistema español tiene una integración institucionalizada de los agentes sociales en los procesos de detección y definición de las cualificaciones profesionales. Sin embargo, la definición del modelo de FP dual se está desarrollando en ausencia de procesos de diálogo social. Además, existen déficits importantes en cuanto a la integración de los sindicatos y los órganos unitarios de representación de los trabajadores al nivel local y de empresa, en claro contraste con los ejemplos de Alemania y Dinamarca (Deissinger y Gonon, 2016; Juul y Helms, 2011; Helms, 2013). En parte debido a estas circunstancias, se está consolidando un discurso sindical crítico y negativo respecto al emergente modelo que puede cuestionar su legitimidad y eficacia.

El análisis de la distribución de las funciones estratégicas y operativas revela que, a pesar de que la regulación concede una autonomía muy limitada a los centros de formación con relación a la elaboración de los currículos y planes educativos, en contraposición al modelo danés (Juul y Helms, 2011), estos disponen en la práctica de bastante libertad para concretar proyectos de formación dual adaptados al entorno productivo local.

Finalmente, se constata que es un modelo que carece de una racionalidad de la agencia definida. Esto se debe a que otorga autonomía a actores autonómicos y locales para concretar distintas aproximaciones de formación dual pero no determina objetivos o resultados que orienten el comportamiento de dichos actores y permitan la evaluación de sus acciones a través de indicadores. 
El análisis realizado muestra la pertinencia de las dimensiones de análisis propuestas para estudiar, clasificar y comparar los sistemas de FP dual, así como su validez para esbozar diagnósticos que puedan servir para mejorar su gobernanza. En este sentido, el artículo ha mostrado que el sistema español de FP dual actual tiene un amplio margen de mejora en el terreno de la gobernanza. Se requeriría mejorar sustancialmente su coordinación, unificando algunos criterios a escala estatal, especialmente en lo tocante a la relación del alumno con la empresa, y creando mecanismos que permitan una coordinación efectiva de las administraciones educativas y laborales y del gobierno central con los autonómicos. También deberían clarificarse los mecanismos de cooperación de los centros y empresas, sin socavar su autonomía. Por otro lado, sería conveniente reforzar en la regulación el papel de los representantes de los trabajadores en la empresa para velar por la calidad de la formación y el cumplimiento de las condiciones laborales establecidas. Finalmente, parece oportuno definir la racionalidad de la agencia. En este caso, es razonable apostar por una opción intermedia entre un modelo orientado hacia resultados y uno basado en el cumplimiento de las normas. Este modelo debiera contar con objetivos fijados a través de procesos de diálogo social, cuyo seguimiento pudiese evaluarse a través de indicadores, en el marco de una regulación más precisa a nivel estatal, en línea con las consideraciones apuntadas anteriormente.

\section{Referencias Bibliográficas}

BUSEMEYER, M.R. Y TRAMPUSCH, C. (2012): The political economy of collective skill formation. (Oxford: Oxford University Press)

CLEMATIDE, B., DAHL, A., VIND, A. Y JØRGENSEN, C. H. (2005): Challenges for the Danish VET system - on the path towards a future model. Berufs- und Wirtschaftspädagogik.

DEISSINGER, T. Y GONON, P. (2016): Stakeholders in the German and Swiss vocational educational and training system: Their role in innovating apprenticeships against the background of academisation, Education + Training, 58 (6), pp.568-577.

EICHHORST, W., RODRÍGUEZ-PLANAS, N., SCHMIDL, R. Y ZIMMERMANN, K. F. (2012): A Roadmap to Vocational Education and Training Systems Around the World, IZA, Discussion Paper Series (http://ftp.iza.org/dp7110.pdf)

FEDERAL INSTITUTE FOR VOCATIONAL EDUCATION AND TRAINING (BIBB) (2011): Vocational Training Regulations and the Process Behind Them (Bonn: BIBB Press).

GARCÍA, M., CASAL, J., MERINO, R. Y SÁNCHEZ, A. (2013): Itinerarios de Abandono Escolar y Transiciones tras la Educación Secundaria Obligatoria, Revista de Educación, mayo-agosto, pp. 65-94.

GREINERT, W-D. (2004): Los sistemas europeos de formación profesional: algunas reflexiones sobre el contexto teórico de su evolución histórica, Revista de Formación Profesional, 32, pp. 18-27. 
HELMS, C. (2013): The role and meaning of vocations in the transition from education to work, International Journal of Training Research 11(2), pp. 166-183.

HIPPACH SCHNEIDER, U. Y HUISMANN, A. (2016). Vocational education and training in Europe - Germany. CEDEFOP ReferNet VET in Europe reports (http://libserver.cedefop.europa.eu/vetelib/2016/2016_CR_DE.pdf=)

HOMS, O. (2008): La formació professional a Espanya. Cap a la societat del coneixement. (Barcelona: Obra Social Fundació La Caixa).

HOOGHE L. Y MARKS G. (2001): Multi-level Governance and European Integration (Lanham: Rowman and Littlefield).

JACOB, M. Y SOLGA, H. (2015): Germany's Vocational Education and Training System in Transformation: Changes in the Participation of Low- and High-Achieving Youth Over Time, European Sociological Review, 31(2): 161-171.

JUUL, I. Y HELMS, C. (2011): Challenges for the dual system and occupational selfgovernance in Denmark, Journal of Vocational Education \& Training 63(3), pp. 289-303.

LOPE, A. Y ALÓS, R. (2013): Las políticas activas de empleo en España: deficientes políticas para malos empleos, Sociología del Trabajo, 77, pp. 92-116.

MARGINSON, P.Y KEUNE, P. (2012): European social dialogue as multi level governance: Towards more autonomy and new dependencies, in Barbier, J.C- (ed) EU Law, Governance and Social Policy European Integration online Papers (EIoP),Special Mini Issue 1, Vol. 16.

MARGINSON P. Y SISSON, K. (2004): European Integration and Industrial Relations (Basingstoke: Palgrave Macmillan).

MARHUENDA, F. (2012): La Formación Profesional (Madrid: Síntesis).

MARISCAL, N. (2003): Teorías políticas de la integración europea (Madrid: Tecnos).

MAYNTZ, R. (2006): Governance Theory als fortentwickelte Steuerungstheorie?, en Schuppert, G.F. (ed.), Governance-Forschung: Vergewisserung über Stand und Entwicklungslinien, pp. 11-20 (Baden-Baden: Nomos).

MENA, A.M. Y MÉNDEZ, J.M. (2009): La técnica del grupo de discusión en investigación cualitativa, Revista Iberoamericana de Educación, 49(3).

MERINO, R. (2009): El sistema de formación profesional en España: tendencias y debates, en GAIRÍN, J., ESSOMBA, M.A. Y MUNTANÉ, D. (eds.): La calidad de la Formación Profesional en Europa, hoy, pp. 131-168 (Bizkaia: Wolters Kluwer).

MERINO, R., GARCÍA, M., CASAL, J. Y SÁNCHEZ, A. (2011): Itinerarios formativos y laborales de los jóvenes graduados en formación profesional. Sobre algunos prejuicios en la formación profesional, Sociología del Trabajo, 72, pp. 137-156.

OLIVER, D. (2010): Complexity in Vocational Education and Training Governance, Research in Comparative and International Education, 5 (3), pp. 261-273. 
PLANAS, J. (2012): ¿Qué es y para qué sirve hoy la formación profesional? De la VT (Formación Profesional) a la VET (Formación y Educación Profesional), Revista de la Asociación de Sociología de la Educación, 5(1), pp.5-15.

RAUNER, F. Y SMITH, E. (2010): Rediscovering Apprenticeship (Dordrecth: Springer).

RAUNER, F. Y WITTING, W. (2010): Differences in the Organisation of Apprenticeship in Europe: findings of a comparative evaluation study, Research in Comparative and International Education, 5, (3), pp. 237-250.

RAUNER, F., WITTING, W. Y DEIMER, L. (2010): Plural Administration in Dual Systems in selected European countries. In Rauner, F.; Smith, E. (eds.): Rediscovering Apprenticeship, pp. 31-43 (Dordrecth: Springer).

ROLLS, S. (2012): Denmark. VET in Europe-Country report. REFERNET. CEDEFOP. (http://www.cedefop.europa.eu/en/publications-and-resources/country-reports/ denmark-vet-europe-country-report-2012)

SALA, G., PLANAS, J. Y VAN ROMPAEY, E. (2010): Especialidad de formación, especialidad de empleo y resultados de inserción. ¿Trabajan los jóvenes donde deberían?, Sociología del Trabajo, 70, pp. 87-106.

SOlGA, H., PROTSCH, P., EBNER, C. Y BRZINSKY-FAY, C. (2014): The German vocational education and training system: Its institutional configuration, strengths and challenges. Discussion Paper SP I 2014-502 October 2014. WZB Berlin Social Science Center.

SPICER, M. (2004): Public administration, the history of ideas, and the reinventing government movement, Public Administration Review, 64 (3), pp. 353-362.

WIBORG, S. Y CORT, P. (2010): The Vocational Education and Training System in Denmark: Continuity and Change, en Gerhard Bosch and Jean Charest (eds.), Vocational Training: international perspectives, pp.84-109 (New York: Routledge). 